International Journal of Pure and Applied Mathematics

Volume $91 \quad$ No. $4 \quad 2014,459-475$

ISSN: 1311-8080 (printed version); ISSN: 1314-3395 (on-line version)

url: http://www.ijpam.eu

doi: http://dx.doi.org/10.12732/ijpam.v91i4.3

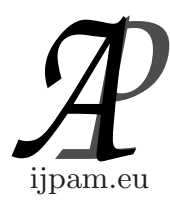

\title{
ON THE THREE STRESS TENSORS FOR LINEARLY ELASTIC CONSTRAINED MATERIALS
}

\author{
Maria Luisa Tonon \\ Department of Mathematics "Giuseppe Peano" \\ University of Turin \\ Via Carlo Alberto 10, Turin, 10123, ITALY
}

\begin{abstract}
In this paper we obtain the constitutive equation for the second Piola-Kirchhoff stress tensor according to the linearized finite theory of elasticity for hyperelastic constrained materials. We show that in such a theory the three stress tensors (Cauchy stress tensor, first and second Piola-Kirchhoff stress tensor) differ by terms that are first order in the strain, while in classical linear theory of elasticity they are indistinguishable to first order of approximation both for unconstrained and constrained materials. Moreover we show that the constitutive equations for the three stress tensors usually adopted in classical linear elasticity are not correct to first order in the strain. Finally we provide an example for a particular material symmetry and for a particular constraint in which the three stress tensors coincide, while in general they are different.
\end{abstract}

AMS Subject Classification: 74B99, 74A10

Key Words: hyperelastic constrained materials, linearized finite elasticity, stress tensors

\section{Introduction}

This paper deals with the constitutive equations for the three stress tensors

Received: October 30, 2013

(c) 2014 Academic Publications, Ltd. url: www.acadpubl.eu 
(Cauchy stress tensor, first and second Piola-Kirchhoff stress tensor) according to the so-called linearized finite theory of elasticity for hyperelastic internally constrained materials (LFTE in the following) formulated by Hoger and Johnson in [3]. In such a theory the constitutive equations are derived by linearization of the corresponding finite constitutive equations with respect to the displacement gradient (see [2], [3], [4] for more details); the constitutive equations which hold in LFTE differ by terms that are first order in the strain from those of the classical linear theory of elasticity for constrained materials (CLTE in the following); for more details concernig CLTE, we refer to [1], Section 58.

Application of LFTE to static or dynamical problems always provides unexpected results; static problems are discussed by Hoger and Johnson in [2], by Marlow in [4] and by Tonon in [7], [8], while wave propagation of acceleration waves is discussed by Tonon in [5], [6]. In general both for static and dynamical problems the results provided by LFTE differ by terms which are first order in the displacement gradient from the corresponding results obtained by CLTE, since only LFTE guarantees the accuracy required by a linear model.

This paper completes [3]: in fact in [3] only the constitutive equations for the Cauchy stress and the first Piola-Kirchhoff stress are derived, while in this paper we also obtain the constitutive expression for the second Piola-Kirchhoff stress, according to LFTE.

It is worth noting that in continuum mechanics the knowledge of a correct constitutive equation for the second Piola-Kirchhoff stress tensor is of primary importance. For brevity, we only recall two applications in which such a tensor plays a fundamental role: the computational mechanics (FEM) and the method due to Green and Spratt to solve boundary-value problems (see [9], Section 67).

Moreover in this paper we compare together the constitutive equations which hold in LFTE for the three stress tensors; finally, following [3] we compare our constitutive equations with those of CLTE for constrained materials.

In Section 2, referring to [3], we briefly summarize the constitutive equation for the Cauchy stress appropriate for LFTE and the field equations used in such a theory. In Section 3 we recall the constitutive equation for the first Piola-Kirchhoff stress given in [3] and we obtain the constitutive equation for the second Piola-Kirchhoff stress. Moreover we show that in LFTE the reaction stresses of the three stress tensors differ by terms that are first order in the strain, while the determinate stresses are the same. Finally we show that the constitutive equations used in CLTE for the three stresses are not correct to first order in the strain. In Section 4 the constitutive equations for the three stresses are specified for incompressible isotropic materials, for inextensible transversely isotropic materials and finally for incompressible transversely isotropic materi- 
als; only in the last case the three stresses are coincidentally indistinguishable. Our constitutive equations are also compared with those provided by CLTE.

\section{The Linearized Finite Theory of Elasticity for hyperelastic constrained materials}

In this section we briefly summarize the field equations of LFTE for hyperelastic constrained materials (see [3]); in particular we recall the constitutive equation for the Cauchy stress appropriate for such a theory. As shown in [3], the constitutive equation for the Cauchy stress usually adopted in CLTE is not correct to first order of approximation.

Denote by $\mathcal{B}=\mathbf{f}\left(\mathcal{B}_{0}\right)$ the deformed configuration of the body, where $\mathcal{B}_{0}$ is the reference configuration and $\mathbf{f}$ is the deformation function; $\mathbf{f}$ carries the point $\mathbf{X} \in \mathcal{B}_{0}$ into the point $\mathbf{x}=\mathbf{f}(\mathbf{X}, \mathbf{t}) \in \mathcal{B}$, where $t$ is the time. We define the displacement $\mathbf{u}$, the deformation gradient $\mathbf{F}$ and the displacement gradient $\mathbf{H}$ as

$$
\begin{gathered}
\mathbf{u}(\mathbf{X}, t)=\mathbf{f}(\mathbf{X}, t)-\mathbf{X}, \\
\mathbf{F}=\operatorname{Grad} \mathbf{f}, \\
\mathbf{H}=\operatorname{Grad} \mathbf{u}=\mathbf{F}-\mathbf{I},
\end{gathered}
$$

where Grad is the gradient operator taken with respect to $\mathbf{X}$, while $\mathbf{I}$ denotes the identity tensor.

The finite Green strain tensor is

$$
\mathbf{E}_{G}=\frac{1}{2}(\mathbf{C}-\mathbf{I}),
$$

where

$$
\mathbf{C}=\mathbf{F}^{T} \mathbf{F}
$$

is the right Cauchy-Green deformation tensor.

For an elastic material subject to a single constraint, the possible strains $\mathbf{E}_{G}$ are restricted by the finite constraint equation

$$
\hat{c}\left(\mathbf{E}_{G}\right)=0 .
$$

If the elastic material is also hyperelastic, we denote by $W=\hat{W}\left(\mathbf{E}_{G}\right)$ the strain energy function.

In finite elasticity for hyperelastic constrained materials the Cauchy stress $\mathbf{T}$ is the sum of the determinate stress $\mathbf{T}_{d}$ and the reaction stress $\mathbf{T}_{r}$, that is

$$
\mathbf{T}=\mathbf{T}_{d}+\mathbf{T}_{r}
$$


where $\mathbf{T}_{d}$ and $\mathbf{T}_{r}$ are defined as follows

$$
\begin{gathered}
\mathbf{T}_{d}=(\operatorname{det} \mathbf{F})^{-1} \mathbf{F} \frac{\partial \hat{W}}{\partial \mathbf{E}_{G}}\left(\mathbf{E}_{G}\right) \mathbf{F}^{T} \\
\mathbf{T}_{r}=q \mathbf{F} \frac{\partial \hat{c}}{\partial \mathbf{E}_{G}}\left(\mathbf{E}_{G}\right) \mathbf{F}^{T},
\end{gathered}
$$

respectively (see [3], formula (3.3)); in (9) $q$ denotes a Lagrange multiplier. Note that in (8), (9) both $\hat{W}\left(\mathbf{E}_{G}\right)$ and $\hat{c}\left(\mathbf{E}_{G}\right)$ are function of the polynomial invariants of the strain appropriate for the material symmetry of the body; denoting by $l\left(\mathbf{E}_{G}\right)$ the complete liste of the polynomial invariants of $\mathbf{E}_{G}$, we have $\hat{W}\left(\mathbf{E}_{G}\right)=\hat{\omega}\left(l\left(\mathbf{E}_{G}\right)\right)$ and $\hat{c}\left(\mathbf{E}_{G}\right)=\hat{\xi}\left(l\left(\mathbf{E}_{G}\right)\right)$.

For isotropy, $l\left(\mathbf{E}_{G}\right)$ is given by

$$
l\left(\mathbf{E}_{G}\right)=\left\{I_{1}, I_{2}, I_{3}\right\}=\left\{\mathbf{I} \cdot \mathbf{E}_{G}, \mathbf{I} \cdot \mathbf{E}_{G}^{2}, \mathbf{I} \cdot \mathbf{E}_{G}^{3}\right\} ;
$$

for transverse isotropy with axis of symmetry $\mathbf{k}, l\left(\mathbf{E}_{G}\right)$ is given by

$$
\begin{aligned}
l\left(\mathbf{E}_{G}\right) & =\left\{I_{1}, I_{2}, I_{3}, I_{4}, I_{5}\right\}= \\
& =\left\{\mathbf{I} \cdot \mathbf{E}_{G}, \mathbf{I} \cdot \mathbf{E}_{G}^{2}, \mathbf{I} \cdot \mathbf{E}_{G}^{3}, \mathbf{k} \cdot \mathbf{E}_{G} \mathbf{k}, \mathbf{k} \cdot \mathbf{E}_{G}^{2} \mathbf{k}\right\} .
\end{aligned}
$$

Now we briefly summarize the method introduced in [3] to linearize constitutive equations (7), (8), (9) with respect to $\mathbf{H}$. By (3), (4), (5) we have

$$
\mathbf{E}_{G}=\mathbf{O}+\frac{1}{2}\left(\mathbf{H}+\mathbf{H}^{T}\right)+o(\mathbf{H}),
$$

where $\mathbf{O}$ is the zero tensor; the tensor $\mathbf{E}$ defined as

$$
\mathbf{E}=\frac{1}{2}\left(\mathbf{H}+\mathbf{H}^{T}\right)
$$

is the infinitesimal strain tensor.

In virtue of (12), for the constraint function $\hat{c}\left(\mathbf{E}_{G}\right)$ in (6) the following expansion holds

$$
\hat{c}\left(\mathbf{E}_{G}\right)=\hat{c}(\mathbf{O})+\frac{1}{2} \frac{\partial \hat{c}}{\partial \mathbf{E}_{G}}(\mathbf{O}) \cdot\left(\mathbf{H}+\mathbf{H}^{T}\right)+o(\mathbf{H}) ;
$$

moreover in (14) we set $\hat{c}(\mathbf{O})=0$, in virtue of (6). Then by combining (6) with (14) we obtain

$$
\frac{1}{2} \frac{\partial \hat{c}}{\partial \mathbf{E}_{G}}(\mathbf{O}) \cdot\left(\mathbf{H}+\mathbf{H}^{T}\right)+o(\mathbf{H})=0,
$$


so that in terms of the infinitesimal strain tensor $\mathbf{E}$ the linearized constraint equation is

$$
\tilde{c}(\mathbf{E})=0
$$

where we have set $\tilde{c}(\mathbf{E})=\frac{\partial \hat{c}}{\partial \mathbf{E}_{G}}(\mathbf{O}) \cdot \mathbf{E}$.

In order to linearize (8) we use for the term $\frac{\partial \hat{W}}{\partial \mathbf{E}_{G}}\left(\mathbf{E}_{G}\right)$ the linearity of the derivative and (12); moreover the requirement that the residual stress vanish provides $\frac{\partial \hat{W}}{\partial \mathbf{E}_{G}}(\mathbf{O})=\mathbf{O}$.

Then for $\frac{\partial \hat{W}}{\partial \mathbf{E}_{G}}\left(\mathbf{E}_{G}\right)$ the following expansion holds

$$
\frac{\partial \hat{W}}{\partial \mathbf{E}_{G}}\left(\mathbf{E}_{G}\right)=\frac{1}{2} \frac{\partial^{2} \hat{W}}{\partial \mathbf{E}_{G} \partial \mathbf{E}_{G}}(\mathbf{O})\left(\mathbf{H}+\mathbf{H}^{T}\right)+o(\mathbf{H}) .
$$

From (3) we have

$$
\operatorname{det} \mathbf{F}=1+\operatorname{tr} \mathbf{H}+o(\mathbf{H})
$$

so that

$$
(\operatorname{det} \mathbf{F})^{-1}=1-\operatorname{tr} \mathbf{H}+o(\mathbf{H}) .
$$

Then by using (3), (17), (19) and discarding terms of order $o(\mathbf{H})$ we obtain the linearization of (8) appropriate for LFTE

$$
\mathbf{T}_{d}=\left.\frac{1}{2} \frac{\partial^{2} \hat{W}}{\partial \mathbf{E}_{G} \partial \mathbf{E}_{G}}(\mathbf{O})\right|_{\tilde{c}}\left(\mathbf{H}+\mathbf{H}^{T}\right)
$$

where the subscript $\tilde{c}$ denotes evaluation on the linearized constraint equation (16) (see [3], formula (3.21)).

Since $\hat{W}\left(\mathbf{E}_{G}\right)=\hat{\omega}\left(l\left(\mathbf{E}_{G}\right)\right)$, the fourth-order tensor $\frac{\partial^{2} \hat{W}}{\partial \mathbf{E}_{G} \partial \mathbf{E}_{G}}(\mathbf{O})$ appearing in (17), (20) can be written explicitly in terms of the polynomial invariants of the strain as follows

$$
\begin{aligned}
& \frac{\partial^{2} \hat{W}}{\partial \mathbf{E}_{G} \partial \mathbf{E}_{G}}(\mathbf{O})=\sum_{p=1}^{n} \sum_{q=1}^{n} \frac{\partial^{2} \hat{\omega}}{\partial I_{p} \partial I_{q}}(l(\mathbf{O})) \frac{\partial I_{p}}{\partial \mathbf{E}_{G}}(\mathbf{O}) \otimes \frac{\partial I_{q}}{\partial \mathbf{E}_{G}}(\mathbf{O})+ \\
& +\sum_{p=1}^{n} \frac{\partial \hat{\omega}}{\partial I_{p}}(l(\mathbf{O})) \frac{\partial^{2} I_{p}}{\partial \mathbf{E}_{G} \partial \mathbf{E}_{G}}(\mathbf{O}),
\end{aligned}
$$


where $n$ is the number of the polynomial invariants and the symbol $\otimes$ denotes tensor product (see [3], formula (3.13)).

In order to linearize (9), we write for the term $\frac{\partial \hat{c}}{\partial \mathbf{E}_{G}}\left(\mathbf{E}_{G}\right)$ the following expansion

$$
\frac{\partial \hat{c}}{\partial \mathbf{E}_{G}}\left(\mathbf{E}_{G}\right)=\frac{\partial \hat{c}}{\partial \mathbf{E}_{G}}(\mathbf{O})+\frac{1}{2} \frac{\partial^{2} \hat{c}}{\partial \mathbf{E}_{G} \partial \mathbf{E}_{G}}(\mathbf{O})\left(\mathbf{H}+\mathbf{H}^{T}\right)+o(\mathbf{H}) .
$$

Then by using (3), (22) and discarding terms of order $o(\mathbf{H})$ we obtain the linearization of (9) appropriate for LFTE

$$
\begin{aligned}
\mathbf{T}_{r}= & q\left\{\frac{\partial \hat{c}}{\partial \mathbf{E}_{G}}(\mathbf{O})+\mathbf{H} \frac{\partial \hat{c}}{\partial \mathbf{E}_{G}}(\mathbf{O})+\frac{\partial \hat{c}}{\partial \mathbf{E}_{G}}(\mathbf{O}) \mathbf{H}^{T}+\right. \\
& \left.+\frac{1}{2} \frac{\partial^{2} \hat{c}}{\partial \mathbf{E}_{G} \partial \mathbf{E}_{G}}(\mathbf{O})\left(\mathbf{H}+\mathbf{H}^{T}\right)\right\}
\end{aligned}
$$

(see [3], formula (3.9)). Since $\hat{c}\left(\mathbf{E}_{G}\right)=\hat{\xi}\left(l\left(\mathbf{E}_{G}\right)\right)$, the derivatives of $\hat{c}$ appearing in (23) can be written explicitly in terms of the polynomial invariants of the strain; for instance, the first derivative takes the form

$$
\frac{\partial \hat{c}}{\partial \mathbf{E}_{G}}(\mathbf{O})=\sum_{p=1}^{n} \frac{\partial \hat{\xi}}{\partial I_{p}}(l(\mathbf{O})) \frac{\partial I_{p}}{\partial \mathbf{E}_{G}}(\mathbf{O}) .
$$

Equations (20), (23) provide the following expression for the Cauchy stress $\mathbf{T}$

$$
\begin{aligned}
\mathbf{T}= & \left.\frac{1}{2} \frac{\partial^{2} \hat{W}}{\partial \mathbf{E}_{G} \partial \mathbf{E}_{G}}(\mathbf{O})\right|_{\tilde{c}}\left(\mathbf{H}+\mathbf{H}^{T}\right)+q\left\{\frac{\partial \hat{c}}{\partial \mathbf{E}_{G}}(\mathbf{O})+\mathbf{H} \frac{\partial \hat{c}}{\partial \mathbf{E}_{G}}(\mathbf{O})+\right. \\
& \left.+\frac{\partial \hat{c}}{\partial \mathbf{E}_{G}}(\mathbf{O}) \mathbf{H}^{T}+\frac{1}{2} \frac{\partial^{2} \hat{c}}{\partial \mathbf{E}_{G} \partial \mathbf{E}_{G}}(\mathbf{O})\left(\mathbf{H}+\mathbf{H}^{T}\right)\right\} .
\end{aligned}
$$

In LFTE the field equations for the deformed configuration $\mathcal{B}$ are given by (3), (13), (16), (24) and the equation of motion

$$
\operatorname{div} \mathbf{T}+\rho \mathbf{b}=\rho \ddot{\mathbf{u}},
$$

where div is the divergence operator taken with respect to $\mathbf{x}, \rho$ and $\mathbf{b}$ are the mass density and the body force density of $\mathcal{B}$, respectively, while the superposed dot denotes time differentiation.

The constitutive equation for $\mathbf{T}$ used in CLTE significantly differs from (24), as shown in [3]. In CLTE it is assumed that $\mathbf{T}$ can be written as

$$
\mathbf{T}=\frac{\partial \tilde{W}_{\tilde{c}}}{\partial \mathbf{E}}(\mathbf{E})+q \frac{\partial \tilde{c}}{\partial \mathbf{E}}(\mathbf{E})
$$


where $\tilde{W}_{\tilde{c}}(\mathbf{E}$ is the quadratic strain energy function for the equivalent unconstrained material that has been evaluated with $\tilde{c}(\mathbf{E})=0$, where $\tilde{c}(\mathbf{E})=0$ is the linear constraint equation (16) (see [3], formula (5.1)).

A detailed comparison between (24) and (26) is given in [3], Section 5; constitutive equation (24) contains some terms dropped in (26), all of which are first order in the strain.

In CLTE such terms disappear since this theory is based on hypotheses of three kinds: the strain energy function is evaluated on the linear constraint equation before differentiation, the constraint function is linearized before the differentiation is carried out and finally the product of the Lagrange multiplier and the deformation gradient is neglected.

\section{The Three Stress Tensors According to the Linearized Finite Theory of Elasticity}

In this section we recall the expression for the first Piola-Kirchhoff stress tensor given in [3] and we obtain the constitutive equation for the second PiolaKirchhoff stress tensor appropriate for LFTE.

Comparison of the expressions for the three stress tensors provided by LFTE shows that they differ by terms that are first order in the strain, while in CLTE the three stress tensors coincide. Moreover we show that the constitutive equations for the three stress tensors usually adopted in CLTE are not correct to first order of approximation.

According to the procedure of linearization exposed in Section 2, the startingpoint to obtain the relations between the first Piola-Kirchhoff stress $\mathbf{S}$ and the Cauchy stress $\mathbf{T}$ or between the second Piola-Kirchhoff stress $\tilde{\mathbf{T}}$ and the Cauchy stress $\mathbf{T}$ are the corresponding relations provided by the finite theory of elasticity.

In finite elasticity $\mathbf{S}$ and $\tilde{\mathbf{T}}$ are defined in terms of $\mathbf{T}$ through the relations

$$
\begin{gathered}
\mathbf{S}=(\operatorname{det} \mathbf{F}) \mathbf{T} \mathbf{F}^{-T} \\
\tilde{\mathbf{T}}=(\operatorname{det} \mathbf{F}) \mathbf{F}^{-1} \mathbf{T} \mathbf{F}^{-T}
\end{gathered}
$$

(see [9], formulas $(43 A .11)_{1},(43 A .11)_{3}$, respectively).

From (3) we have $\mathbf{F}=\mathbf{I}+\mathbf{H}$; then the following expansions hold

$$
\begin{gathered}
\mathbf{F}^{-1}=\mathbf{I}-\mathbf{H}+o(\mathbf{H}) \\
\mathbf{F}^{-T}=\mathbf{I}-\mathbf{H}^{T}+o(\mathbf{H}) .
\end{gathered}
$$


By using (18), (29), (30) and discarding terms of order $o(\mathbf{H})$, relations (27), (28) become

$$
\begin{gathered}
\mathbf{S}=\mathbf{T}+(\operatorname{tr} \mathbf{H}) \mathbf{T}-\mathbf{T} \mathbf{H}^{T} \\
\tilde{\mathbf{T}}=\mathbf{T}+(\operatorname{tr} \mathbf{H}) \mathbf{T}-\mathbf{H T}-\mathbf{T} \mathbf{H}^{T},
\end{gathered}
$$

respectively.

Then the explicit expressions for $\mathbf{S}$ and $\tilde{\mathbf{T}}$ in LFTE can be obtained by substituting (24) into (31), (32), respectively; of course after substitution only terms that are linear in $\mathbf{H}$ must be retained.

This procedure of linearization provides for $\mathbf{S}$ and $\tilde{\mathbf{T}}$ the following expressions

$$
\begin{aligned}
\mathbf{S}= & \left.\frac{1}{2} \frac{\partial^{2} \hat{W}}{\partial \mathbf{E}_{G} \partial \mathbf{E}_{G}}(\mathbf{O})\right|_{\tilde{c}}\left(\mathbf{H}+\mathbf{H}^{T}\right)+q\left\{(1+\operatorname{tr} \mathbf{H}) \frac{\partial \hat{c}}{\partial \mathbf{E}_{G}}(\mathbf{O})+\right. \\
& \left.+\mathbf{H} \frac{\partial \hat{c}}{\partial \mathbf{E}_{G}}(\mathbf{O})+\frac{1}{2} \frac{\partial^{2} \hat{c}}{\partial \mathbf{E}_{G} \partial \mathbf{E}_{G}}(\mathbf{O})\left(\mathbf{H}+\mathbf{H}^{T}\right)\right\}
\end{aligned}
$$

and

$$
\begin{aligned}
\tilde{\mathbf{T}}= & \left.\frac{1}{2} \frac{\partial^{2} \hat{W}}{\partial \mathbf{E}_{G} \partial \mathbf{E}_{G}}(\mathbf{O})\right|_{\tilde{c}}\left(\mathbf{H}+\mathbf{H}^{T}\right)+q\left\{(1+\operatorname{tr} \mathbf{H}) \frac{\partial \hat{c}}{\partial \mathbf{E}_{G}}(\mathbf{O})+\right. \\
& \left.+\frac{1}{2} \frac{\partial^{2} \hat{c}}{\partial \mathbf{E}_{G} \partial \mathbf{E}_{G}}(\mathbf{O})\left(\mathbf{H}+\mathbf{H}^{T}\right)\right\}
\end{aligned}
$$

respectively (for constitutive equation (33), (see [3], formula (3.23)).

Moreover note that in LFTE the tensor $\tilde{\mathbf{T}}$ is symmetric, while $\mathbf{S}$ is not symmetric; the same occurs in finite theory of elasticity. In this sense LFTE retains the "memory"' of the finite theory of elasticity.

Finally, for the sake of completeness we obtain the other relations involving $\mathbf{T}, \mathbf{S}, \tilde{\mathbf{T}}$ within the framework of LFTE. The starting-point are the relations which hold in finite elasticity, that is

$$
\begin{gathered}
\tilde{\mathbf{T}}=\mathbf{F}^{-1} \mathbf{S} \\
\mathbf{T}=(\operatorname{det} \mathbf{F})^{-1} \mathbf{S} \mathbf{F}^{T} \\
\mathbf{T}=(\operatorname{det} \mathbf{F})^{-1} \mathbf{F} \tilde{\mathbf{T}} \mathbf{F}^{T} \\
\mathbf{S}=\mathbf{F} \tilde{\mathbf{T}} ;
\end{gathered}
$$

by using (29), (19) and the procedure of linearization exposed above, in LFTE relations (35), (36), (37), (38) become

$$
\tilde{\mathbf{T}}=\mathbf{S}-\mathbf{H S}
$$




$$
\begin{gathered}
\mathbf{T}=\mathbf{S}-(\operatorname{tr} \mathbf{H}) \mathbf{S}+\mathbf{S H}^{T} \\
\mathbf{T}=\tilde{\mathbf{T}}-(\operatorname{tr} \mathbf{H}) \tilde{\mathbf{T}}+\mathbf{H} \tilde{\mathbf{T}}+\tilde{\mathbf{T}} \mathbf{H}^{T} \\
\mathbf{S}=\tilde{\mathbf{T}}+\mathbf{H} \tilde{\mathbf{T}}
\end{gathered}
$$

respectively.

Now we devote our attention to the comparison of expressions (24), (33), (34) obtained for the three stress tensors: we see that the determinate stress is the same, while the reaction stress is different, so that we can claim that in LFTE the three stress tensors differ by terms that are first order in the strain.

Constitutive equations (24), (33), (34) are fundamentally different from the corresponding equations used in CLTE. In such a theory the three stress tensors are indistinguishable to this order of approximation $(\mathbf{T}=\mathbf{S}=\tilde{\mathbf{T}})$ both for unconstrained and constrained materials; they become distinguishable only if quadratic terms in the deformation gradient are taken into account.

\section{Examples}

In this section the general constitutive equations for $\mathbf{T}, \mathbf{S}, \tilde{\mathbf{T}}$ obtained in Section 3 are specified for incompressible isotropic materials, for inextensible transversely isotropic materials and finally for incompressible transversely isotropic materials. For incompressible transversely isotropic materials the constitutive equations provided by LFTE for the three stress tensors coincide. Our constitutive equations are also compared with the corresponding equations used in CLTE.

(i) Incompressible isotropic materials

For the constraint of incompressibility, equations (6), (16) become

$$
\operatorname{det}\left(2 \mathbf{E}_{G}+\mathbf{I}\right)-1=0
$$

and

$$
\operatorname{tr} \mathbf{E}=0,
$$

respectively.

For isotropy, the complete list of the polynomial invariants of $\mathbf{E}_{G}$ is given by (10), so that the first derivatives of the invariants are

$$
\frac{\partial I_{1}}{\partial \mathbf{E}_{G}}=\mathbf{I}, \quad \frac{\partial I_{2}}{\partial \mathbf{E}_{G}}=2 \mathbf{E}_{G}, \quad \frac{\partial I_{3}}{\partial \mathbf{E}_{G}}=3 \mathbf{E}_{G}^{2} .
$$


By (45) the second derivatives of the invariants, written in component form, are

$$
\begin{aligned}
\frac{\partial^{2} I_{1}}{\partial\left(\mathbf{E}_{G}\right)_{i j} \partial\left(\mathbf{E}_{G}\right)_{k l}}= & 0 \\
\frac{\partial^{2} I_{2}}{\partial\left(\mathbf{E}_{G}\right)_{i j} \partial\left(\mathbf{E}_{G}\right)_{k l}}= & \delta_{i k} \delta_{j l}+\delta_{i l} \delta_{j k} \\
\frac{\partial^{2} I_{3}}{\partial\left(\mathbf{E}_{G}\right)_{i j} \partial\left(\mathbf{E}_{G}\right)_{k l}}= & \frac{3}{2}\left\{\delta_{i k}\left(\mathbf{E}_{G}\right)_{j l}+\delta_{l j}\left(\mathbf{E}_{G}\right)_{k i}+\right. \\
& \left.+\delta_{k j}\left(\mathbf{E}_{G}\right)_{i l}+\delta_{l i}\left(\mathbf{E}_{G}\right)_{k j}\right\} .
\end{aligned}
$$

Substitution of (45), (46) into (21) provides for the components of the tensor $\frac{\partial^{2} \hat{W}}{\partial \mathbf{E}_{G} \partial \mathbf{E}_{G}}(\mathbf{O})$ the expression

$$
\frac{\partial^{2} \hat{W}}{\partial\left(\mathbf{E}_{G}\right)_{i j} \partial\left(\mathbf{E}_{G}\right)_{k l}}(\mathbf{O})=\lambda \delta_{i j} \delta_{k l}+\mu\left(\delta_{i k} \delta_{j l}+\delta_{i l} \delta_{j k}\right),
$$

where $\lambda$ and $\mu$ are the Lamé moduli defined as follows

$$
\begin{aligned}
\lambda & =\frac{\partial^{2} \hat{\omega}}{\partial I_{1} \partial I_{1}}(l(\mathbf{O})) \\
\mu & =\frac{\partial \hat{\omega}}{\partial I_{2}}(l(\mathbf{O})) .
\end{aligned}
$$

Moreover, from (43) we have

$$
\begin{aligned}
& \frac{\partial \hat{c}}{\partial\left(\mathbf{E}_{G}\right)_{i j}}(\mathbf{O})=\delta_{i j} \\
& \frac{\partial^{2} \hat{c}}{\partial\left(\mathbf{E}_{G}\right)_{i j} \partial\left(\mathbf{E}_{G}\right)_{k l}}(\mathbf{O})=-2 \delta_{i k} \delta_{j l}-2 \delta_{i l} \delta_{j k}+4 \delta_{i j} \delta_{k l} .
\end{aligned}
$$

By substituting (47), (49) into (24), (33), (34) and using (44) we obtain the following expressions for the three stress tensors

$$
\begin{gathered}
\mathbf{T}=\mu\left(\mathbf{H}+\mathbf{H}^{T}\right)+q \mathbf{I} \\
\mathbf{S}=\mu\left(\mathbf{H}+\mathbf{H}^{T}\right)+q\left(\mathbf{I}-\mathbf{H}^{T}\right) \\
\tilde{\mathbf{T}}=\mu\left(\mathbf{H}+\mathbf{H}^{T}\right)+q\left(\mathbf{I}-\mathbf{H}-\mathbf{H}^{T}\right)
\end{gathered}
$$


(for $\mathbf{T}$ and $\mathbf{S}$, see [3], formulas (4.15), (4.16). Though the final formulas for the tensors $\mathbf{T}$ and $\mathbf{S}$ obtained in [3] are correct, in such a paper some mistakes occur in the formulas which provide the second derivatives of the polynomial invariants and the second derivatives of $\hat{W}$ and $\hat{c}$ ).

In CLTE the Cauchy stress tensor $\mathbf{T}$ is given by (26). The linear constraint equation (44) provides

$$
\frac{\partial \tilde{c}}{\partial \mathbf{E}}=\mathbf{I}
$$

since for a linearly elastic isotropic material the strain energy function is

$$
\tilde{W}(\mathbf{E})=\lambda(\operatorname{tr} \mathbf{E})^{2}+\mu \operatorname{tr}\left(\mathbf{E}^{2}\right),
$$

we have

$$
\tilde{W}_{\tilde{c}}(\mathbf{E})=\mu \operatorname{tr}\left(\mathbf{E}^{2}\right)
$$

and

$$
\frac{\partial \tilde{W}_{\tilde{c}}}{\partial \mathbf{E}}(\mathbf{E})=2 \mu \mathbf{E} .
$$

Then in this particular case equation (26) reduces to (50), so that the constitutive equation for $\mathbf{T}$ used in CLTE is coincidentally correct.

Moreover CLTE requires $\mathbf{S}=\tilde{\mathbf{T}}=\mathbf{T}$, since in such a theory the terms involving a product of the Lagrange multiplier and the deformation gradient are neglected, while in (51), (52) they are retained; then in virtue of (51), (52) the expressions for $\mathbf{S}$ and $\tilde{\mathbf{T}}$ usually adopted in CLTE are not correct to first order in the strain.

(ii) Inextensible transversely isotropic materials

For the constraint of inextensibility, denoting by $\mathbf{k}$ the axis of inextensibility, equations (6), (16) take the form

$$
(\mathbf{k} \otimes \mathbf{k}) \cdot \mathbf{E}_{G}=0
$$

and

$$
(\mathbf{k} \otimes \mathbf{k}) \cdot \mathbf{E}=0,
$$

respectively.

For a transversely isotropic material whose axis of symmetry coincides with the axis of inextensibility $\mathbf{k}$, the complete list of the polynomial invariants of $\mathbf{E}_{G}$ is given by (11).

The first derivatives of the five invariants are 


$$
\begin{aligned}
\frac{\partial I_{1}}{\partial \mathbf{E}_{G}} & =\mathbf{I} \\
\frac{\partial I_{2}}{\partial \mathbf{E}_{G}} & =2 \mathbf{E}_{G} \\
\frac{\partial I_{3}}{\partial \mathbf{E}_{G}} & =3 \mathbf{E}_{G}^{2} \\
\frac{\partial I_{4}}{\partial \mathbf{E}_{G}} & =\mathbf{k} \otimes \mathbf{k} \\
\frac{\partial I_{5}}{\partial \mathbf{E}_{G}} & =\mathbf{k} \otimes\left(\mathbf{E}_{G} \mathbf{k}\right)+\left(\mathbf{E}_{G} \mathbf{k}\right) \otimes \mathbf{k} .
\end{aligned}
$$

By (59) the second derivatives of the five invariants, written in component form, are

$$
\begin{aligned}
\frac{\partial^{2} I_{1}}{\partial\left(\mathbf{E}_{G}\right)_{i j} \partial\left(\mathbf{E}_{G}\right)_{k l}}= & 0 \\
\frac{\partial^{2} I_{2}}{\partial\left(\mathbf{E}_{G}\right)_{i j} \partial\left(\mathbf{E}_{G}\right)_{k l}}= & \delta_{i k} \delta_{j l}+\delta_{i l} \delta_{j k} \\
\frac{\partial^{2} I_{3}}{\partial\left(\mathbf{E}_{G}\right)_{i j} \partial\left(\mathbf{E}_{G}\right)_{k l}}= & \frac{3}{2}\left\{\delta_{i k}\left(\mathbf{E}_{G}\right)_{j l}+\delta_{l j}\left(\mathbf{E}_{G}\right)_{k i}+\right. \\
& \left.+\delta_{k j}\left(\mathbf{E}_{G}\right)_{i l}+\delta_{l i}\left(\mathbf{E}_{G}\right)_{k j}\right\} \\
\frac{\partial^{2} I_{4}}{\partial\left(\mathbf{E}_{G}\right)_{i j} \partial\left(\mathbf{E}_{G}\right)_{k l}}= & 0 \\
\frac{\partial^{2} I_{5}}{\partial\left(\mathbf{E}_{G}\right)_{i j} \partial\left(\mathbf{E}_{G}\right)_{k l}}= & \frac{1}{2}\left(k_{i} k_{l} \delta_{j k}+k_{i} k_{k} \delta_{j l}+k_{j} k_{l} \delta_{i k}+k_{j} k_{k} \delta_{l i}\right) .
\end{aligned}
$$

Substitution of (59), (60) into (21) provides for the components of the tensor $\frac{\partial^{2} \hat{W}}{\partial \mathbf{E}_{G} \partial \mathbf{E}_{G}}(\mathbf{O})$ the following expression

$$
\begin{aligned}
& \frac{\partial^{2} \hat{W}}{\partial\left(\mathbf{E}_{G}\right)_{i j} \partial\left(\mathbf{E}_{G}\right)_{k l}}(\mathbf{O})=\alpha_{1} \delta_{i j} \delta_{k l}+\alpha_{2}\left(\delta_{i j} k_{k} k_{l}+k_{i} k_{j} \delta_{k l}\right)+ \\
& \quad+\alpha_{3} k_{i} k_{j} k_{k} k_{l}+\alpha_{4}\left(\delta_{i k} \delta_{j l}+\delta_{i l} \delta_{j k}\right)+\frac{1}{2} \alpha_{5}\left(k_{i} k_{l} \delta_{j k}+\right. \\
& \left.\quad+k_{i} k_{k} \delta_{j l}+k_{j} k_{l} \delta_{i k}+k_{j} k_{k} \delta_{i l}\right)
\end{aligned}
$$


where the coefficients $\alpha_{1}, \alpha_{2}, \alpha_{3}, \alpha_{4}, \alpha_{5}$ are defined as follows

$$
\begin{aligned}
\alpha_{1} & =\frac{\partial^{2} \hat{\omega}}{\partial I_{1} \partial I_{1}}(l(\mathbf{O})) \\
\alpha_{2} & =\frac{\partial^{2} \hat{\omega}}{\partial I_{1} \partial I_{4}}(l(\mathbf{O})) \\
\alpha_{3} & =\frac{\partial^{2} \hat{\omega}}{\partial I_{4} \partial I_{4}}(l(\mathbf{O})) \\
\alpha_{4} & =\frac{\partial \hat{\omega}}{\partial I_{2}}(l(\mathbf{O})) \\
\alpha_{5} & =\frac{\partial \hat{\omega}}{\partial I_{5}}(l(\mathbf{O})) .
\end{aligned}
$$

From (57) we have

$$
\begin{aligned}
& \frac{\partial \hat{c}}{\partial\left(\mathbf{E}_{G}\right)_{i j}}(\mathbf{O})=k_{i} k_{j} \\
& \frac{\partial^{2} \hat{c}}{\partial\left(\mathbf{E}_{G}\right)_{i j} \partial\left(\mathbf{E}_{G}\right)_{k l}}(\mathbf{O})=0 .
\end{aligned}
$$

By substituting (61), (63) into (24) and using (58) we obtain for the Cauchy stress tensor $\mathbf{T}$ the form

$$
\begin{aligned}
\mathbf{T}= & \alpha_{1}(\operatorname{tr} \mathbf{H}) \mathbf{I}+\alpha_{2}(\operatorname{tr} \mathbf{H}) \mathbf{k} \otimes \mathbf{k}+\alpha_{4}\left(\mathbf{H}+\mathbf{H}^{T}\right)+ \\
& +\frac{1}{2} \alpha_{5}\left\{\mathbf{H}(\mathbf{k} \otimes \mathbf{k})+\mathbf{H}^{T}(\mathbf{k} \otimes \mathbf{k})+(\mathbf{k} \otimes \mathbf{k}) \mathbf{H}+\right. \\
& \left.+(\mathbf{k} \otimes \mathbf{k}) \mathbf{H}^{T}\right\}+q\left\{\mathbf{k} \otimes \mathbf{k}+\mathbf{H}(\mathbf{k} \otimes \mathbf{k})+(\mathbf{k} \otimes \mathbf{k}) \mathbf{H}^{T}\right\}
\end{aligned}
$$

The constitutive equations for the first and the second Piola-Kirchhoff stress tensor are given by (33), (34), respectively; with the use of (58), (61), (63) they take the form

$$
\begin{aligned}
\mathbf{S}= & \alpha_{1}(\operatorname{tr} \mathbf{H}) \mathbf{I}+\alpha_{2}(\operatorname{tr} \mathbf{H}) \mathbf{k} \otimes \mathbf{k}+\alpha_{4}\left(\mathbf{H}+\mathbf{H}^{T}\right)+ \\
& +\frac{1}{2} \alpha_{5}\left\{\mathbf{H}(\mathbf{k} \otimes \mathbf{k})+\mathbf{H}^{T}(\mathbf{k} \otimes \mathbf{k})+(\mathbf{k} \otimes \mathbf{k}) \mathbf{H}+\right. \\
& \left.+(\mathbf{k} \otimes \mathbf{k}) \mathbf{H}^{T}\right\}+q\{\mathbf{k} \otimes \mathbf{k}+\mathbf{H}(\mathbf{k} \otimes \mathbf{k})+(\operatorname{tr} \mathbf{H}) \mathbf{k} \otimes \mathbf{k}\}
\end{aligned}
$$




$$
\begin{aligned}
\tilde{\mathbf{T}}= & \alpha_{1}(\operatorname{tr} \mathbf{H}) \mathbf{I}+\alpha_{2}(\operatorname{tr} \mathbf{H}) \mathbf{k} \otimes \mathbf{k}+\alpha_{4}\left(\mathbf{H}+\mathbf{H}^{T}\right)+ \\
& +\frac{1}{2} \alpha_{5}\left\{\mathbf{H}(\mathbf{k} \otimes \mathbf{k})+\mathbf{H}^{T}(\mathbf{k} \otimes \mathbf{k})+(\mathbf{k} \otimes \mathbf{k}) \mathbf{H}+\right. \\
& \left.+(\mathbf{k} \otimes \mathbf{k}) \mathbf{H}^{T}\right\}+q\{\mathbf{k} \otimes \mathbf{k}+(\operatorname{tr} \mathbf{H}) \mathbf{k} \otimes \mathbf{k}\}
\end{aligned}
$$

(for $\mathbf{T}$ and $\mathbf{S}$, see [3], formulas (4.27), (4.28). Also for this example we note that the formulas obtained in [3] for $\mathbf{T}$ and $\mathbf{S}$ are correct, but in such a paper the expressions written for the second derivatives of the polynomial invariants and for the second derivatives of $\hat{W}$ contain some mistakes).

In CLTE the stress tensor $\mathbf{T}$ is given by (26). The linear constraint equation (58) provides

$$
\frac{\partial \tilde{c}}{\partial \mathbf{E}}(\mathbf{E})=\mathbf{k} \otimes \mathbf{k}
$$

For a transversely isotropic material the strain energy function is

$$
\begin{aligned}
\tilde{W}(\mathbf{E}) & =\beta_{1}(\operatorname{tr} \mathbf{E})^{2}+\beta_{2} \operatorname{tr}\left(\mathbf{E}^{2}\right)+\beta_{3}(\mathbf{k} \otimes \mathbf{k}) \cdot \mathbf{E}^{2}+ \\
& +\beta_{4}\{(\mathbf{k} \otimes \mathbf{k}) \cdot \mathbf{E}\}^{2}+\beta_{5}(\operatorname{tr} \mathbf{E})\{(\mathbf{k} \otimes \mathbf{k}) \cdot \mathbf{E}\}
\end{aligned}
$$

in virtue of constraint equation (58), (68) reduces to

$$
\tilde{W}_{\tilde{c}}(\mathbf{E})=\beta_{1}(\operatorname{tr} \mathbf{E})^{2}+\beta_{2} \operatorname{tr}\left(\mathbf{E}^{2}\right)+\beta_{3}(\mathbf{k} \otimes \mathbf{k}) \cdot \mathbf{E}^{2}
$$

therefore

$$
\frac{\partial \tilde{W}_{\tilde{c}}}{\partial \mathbf{E}}(\mathbf{E})=2 \beta_{1}(\operatorname{tr} \mathbf{E}) \mathbf{I}+2 \beta_{2} \mathbf{E}+\beta_{3}\{(\mathbf{k} \otimes \mathbf{k}) \mathbf{E}+\mathbf{E}(\mathbf{k} \otimes \mathbf{k})\} .
$$

By substituting (67), (70) into (26) we obtain for the tensor $\mathbf{T}$ the form

$$
\mathbf{T}=2 \beta_{1}(\operatorname{tr} \mathbf{E}) \mathbf{I}+2 \beta_{2} \mathbf{E}+\beta_{3}\{(\mathbf{k} \otimes \mathbf{k}) \mathbf{E}+\mathbf{E}(\mathbf{k} \otimes \mathbf{k})\}+q \mathbf{k} \otimes \mathbf{k},
$$

usually adopted in CLTE.

Comparison of (64) with (71) shows that in CLTE both the determinate stress and the reaction stress are not correct to first order in the strain. The same holds for $\mathbf{S}$ and $\tilde{\mathbf{T}}$.

(iii) Incompressible transversely isotropic materials

In this case (61), (62) hold, while the linear constraint equation given by (44) provides (49). 
By substituting (49), (61) into (24) and using (44) we have for the stress T the following form

$$
\begin{aligned}
\mathbf{T} & =\frac{1}{2} \alpha_{2}\left\{(\mathbf{k} \otimes \mathbf{k}) \cdot\left(\mathbf{H}+\mathbf{H}^{T}\right)\right\} \mathbf{I}+ \\
& +\frac{1}{2} \alpha_{3}\left\{(\mathbf{k} \otimes \mathbf{k}) \cdot\left(\mathbf{H}+\mathbf{H}^{T}\right)\right\} \mathbf{k} \otimes \mathbf{k}+\alpha_{4}\left(\mathbf{H}+\mathbf{H}^{T}\right)+ \\
& +\frac{1}{2} \alpha_{5}\left\{(\mathbf{k} \otimes \mathbf{k}) \mathbf{H}+(\mathbf{k} \otimes \mathbf{k}) \mathbf{H}^{T}+\mathbf{H}(\mathbf{k} \otimes \mathbf{k})+\right. \\
& \left.+\mathbf{H}^{T}(\mathbf{k} \otimes \mathbf{k})\right\}+q \mathbf{I} .
\end{aligned}
$$

Moreover, by substituting (49), (61) into (33), (34) and using (44) we obtain both for $\mathbf{S}$ and $\tilde{\mathbf{T}}$ expression (72). Then we can claim that in this particular case the constitutive equations provided by LFTE for the three stress tensors coincide.

In order to compare our expressions for the three stress tensors with the corresponding expressions in CLTE we substitute the linear constraint equation (44) into the strain energy function (68); then (68) reduces to

$$
\tilde{W}_{\tilde{c}}(\mathbf{E})=\beta_{2} \operatorname{tr}\left(\mathbf{E}^{2}\right)+\beta_{3}(\mathbf{k} \otimes \mathbf{k}) \cdot \mathbf{E}^{2}+\beta_{4}\{(\mathbf{k} \otimes \mathbf{k}) \cdot \mathbf{E}\}^{2} .
$$

Therefore

$$
\begin{aligned}
\frac{\partial \tilde{W}_{\tilde{c}}}{\partial \mathbf{E}}(\mathbf{E})= & 2 \beta_{2} \mathbf{E}+\beta_{3}\{(\mathbf{k} \otimes \mathbf{k}) \mathbf{E}+\mathbf{E}(\mathbf{k} \otimes \mathbf{k})\}+ \\
& +2 \beta_{4}\{(\mathbf{k} \otimes \mathbf{k}) \cdot \mathbf{E}\} \mathbf{k} \otimes \mathbf{k}
\end{aligned}
$$

Substitution of (53), (74) into (26) provides for the tensor $\mathbf{T}$ in CLTE the form

$$
\begin{aligned}
\mathbf{T}= & 2 \beta_{2} \mathbf{E}+\beta_{3}\{(\mathbf{k} \otimes \mathbf{k}) \mathbf{E}+\mathbf{E}(\mathbf{k} \otimes \mathbf{k})\}+ \\
& +2 \beta_{4}\{(\mathbf{k} \otimes \mathbf{k}) \cdot \mathbf{E}\} \mathbf{k} \otimes \mathbf{k}+q \mathbf{I} .
\end{aligned}
$$

If we compare (72) with (75), we see that the expression for the determinate stress obtained in CLTE is not correct to first order of approximation. In fact, the first term in the determinate stress appearing in (72) is missed in (75), while the other terms coincide; the first term is missed because in CLTE the constraint equation is linearized before the differentiation is carried out. 


\section{Conclusions}

In this paper by using the linearized finite theory of elasticity we obtain the constitutive equation for the second Piola-Kirchhoff stress tensor appropriate for constrained hyperelastic materials. Moreover we show that to first order of approximation in such a theory the three stress tensors are different, while in classical linear theory of elasticity they are indistinguishable.

For the moment we stop here our analysis; nevertheless it is worth noting that the results obtained in this paper represent the starting-point in order to derive correct expansions up to terms of second order in the displacement gradient for the three stress tensors in constrained hyperelastic materials. We leave this study for our next paper.

\section{Acknowledgements}

This research was supported by the Department of Mathematics "Giuseppe Peano" of the University of Turin (Italy).

\section{References}

[1] M.E. Gurtin, The Linear Theory of Elasticity, Handbuch der Physik, VI a/2, Springer, Germany (1972), doi: 10.1007/978-3-642-69567-4_1.

[2] A. Hoger, B.E. Johnson, Linear elasticity for constrained materials: Incompressibility, J. Elasticity, 38 (1995), 69-93, doi: 10.1007/BF00121464.

[3] A. Hoger, B.E. Johnson, Linear elasticity for constrained materials: General theory for hyperelasticity, J. Elasticity, 38 (1995), 95-120, doi: 10.1007/BF00121465.

[4] R.S. Marlow, On the stress in an internally constrained elastic material, J. Elasticity, 27 (1992), 97-131, doi: 10.1007/BF00041645.

[5] M.L. Tonon, Waves in constrained linear elastic materials, J. Elasticity, 69 (2002), 15-39, doi: 10.1023/A:1027334213793.

[6] M.L. Tonon, Wave propagation according to the linearized finite theory of elasticity, International J. of Pure and Applied Mathematics, 40, No.1 (2007), 135-152. 
[7] M.L. Tonon, A note on the linearized finite theory of elasticity, International J. of Pure and Applied Mathematics, 58, No. 2 (2010), 195-208.

[8] M.L. Tonon, On simple shear for incompressible isotropic linear elastic materials, International J. of Pure and Applied Mathematics, 75, No. 3 (2012), 353-370.

[9] C. Truesdell, W. Noll, The Non-Linear Field Theories of Mechanics, Handbuch der Physik, III/3, Springer, Germany (1965). 
